\title{
Peripheral Display for Conveying Real-time Pain Condition of Persons with Severe Intellectual Disabilities to their Caregivers
}

\author{
Zhiping Zhang \\ Department of Industrial Design, \\ Eindhoven University of Technology \\ arya.zhangpika@gmail.com
}

\author{
Helen Korving* \\ Department of Industrial Design, \\ Eindhoven University of Technology \\ \& Department of Clinical Child and \\ Family Studies, Vrije Universiteit \\ h.korving@tue.nl
}

\author{
Paula Sterkenburg ${ }^{\dagger}$ \\ Department of Clinical Child and \\ Family Studies, Vrije Universiteit \\ Amsterdam \& Bartiméus, Doorn \\ p.s.sterkenburg@vu.nl
}

\author{
Loe Feijs $\ddagger$ \\ Department of Industrial Design, \\ Eindhoven University of Technology \\ l.m.g.feijs@tue.nl
}

\author{
Emilia Barakova§ \\ Department of Industrial Design, \\ Eindhoven University of Technology \\ e.i.barakova@tue.nl
}

\begin{abstract}
An effective assisting tool for caregivers to monitor pain of persons with severe intellectual disabilities (SID) is eagerly needed, since these persons have difficulties with self-report. The Bio response System detecting stress and the techniques with potential to distinguish pain from stress suggest the possibility to detect pain with physiological data. In the current paper, we propose the design of a peripheral display for making caregivers aware of the real-time pain condition of their clients without added attention burden. An iterative user-centered design process resulted in two prototypes and corresponding evaluations of one peripheral display. The potential of the display to help caregivers be promptly aware of the pain condition of clients was tested with targeted users. Guidelines for the design of peripheral display applications in similar semihospitalized contexts are provided. Further steps in this study will be to test the adjusted Bio response System for detecting pain in persons with SID adequately.
\end{abstract}

\section{CCS CONCEPTS}

-Human-centered computing; • Visulization; • Visualization design and evaluation methods;

\section{KEYWORDS}

Peripheral Display, Pain Detection, Electrodermal Activity (EDA) Measurements, Situation Awareness, User-centered Interface

\section{ACM Reference Format:}

Zhiping Zhang, Helen Korving, Paula Sterkenburg, Loe Feijsł; and Emilia Barakova§. 2021. Peripheral Display for Conveying Real-time Pain Condition of Persons with Severe Intellectual Disabilities to their Caregivers.

\footnotetext{
${ }^{*}$ Corresponding author.

${ }^{\dagger}$ Corresponding author.
}

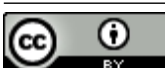

This work is licensed under a Creative Commons Attribution International 4.0 License.

Chinese CHI 2021, October 16, 17, 2021, Online, Hong Kong

(c) 2021 Copyright held by the owner/author(s).

ACM ISBN 978-1-4503-8695-1/21/10.

https://doi.org/10.1145/3490355.3490362
In The Ninth International Symposium of Chinese CHI (Chinese CHI 2021), October 16, 17, 2021, Online, Hong Kong. ACM, New York, NY, USA, 9 pages. https://doi.org/10.1145/3490355.3490362

\section{INTRODUCTION}

\subsection{Pain Detection and Bio response System}

Pain detection among persons with severe intellectual disability (SID) is usually a challenge for caregivers because of clients' subtle and mostly non-verbal expressive behavior [11, 23, 25, 27]. This vulnerable group of people may suffer from pain on a daily basis, but their pain is not always actively managed [23]. Previous research has shown the far-reaching consequences, such as self-harm and problem behavior, caused by insensitive responses or managements of the pain of persons with SID [3, 6, 22]. At the same time, research and methods for helping caregivers to detect pain among non-verbally communicating intellectually disabled persons is still scarce [19]. At the 2017 Dutch national "NetWork" meetings for the ZonMw-project "Social relationships and ICT", the need to have better understandings in the pain related behavior of children and adults with SID has been emphasized by both parents and caregivers. A more effective, objective and accurate way to detect pain in those with SID and to communicate it to caregivers is needed. To help caregivers respond more sensitive and accurately towards the emotions of persons with SID, a Bio response System was developed to detect a SID- client's stress through physiological data [17]. In this Bio response System, a "Smart Sock" with sensors detecting skin conductance and other physiological data (i.e. heart rate, and heart rate variability) was developed with the intent to measure arousal in the wearer [17, 24]. In addition, an accompanying Android application was designed and implemented as part of the system, to show the amount of arousal to caregivers [17, 24]. This system has already been used in several studies on the measurement and visualization of arousal [9, 24, 28]. This arousal app is intended to help caregivers or parents to notice, understand and attune to the emotional responses in persons with SID and lead to smoother communication.

Although the Bio response System was not used to detect pain in previous studies, there are indications in the literature that, with the adjustments in the processing of data, the system can be used 
to detect skin conductance variability (SCV). It has been shown that SCV can be used to detect pain in persons with SID [1]. In the study's current stage, we are searching for an adequate graphical and semantic design of a future 'Pain App' so it can properly communicate pain intensity to the caregiver, to also represent context, and peripheral and undisturbed awareness.

Technology can help relieve caregivers' stress and burden, but only if the right technology is used in the right way. The developed application is meant for conveying information and therefore should be adjusted to correspond to the context changes from stress detection into pain detection. For example, pain is a different experience than arousal and should semantically be alternatively represented. Furthermore, the arousal app was designed to convey a single subject's real-time information, while normally several clients live together in care homes and one caregiver is responsible for multiple clients. Therefore, the aim of this project is to design a better way to convey real-time and multi-client pain information in a seamless manner. The "SID Pain APP" is being developed to help communicate the real-time multi-client pain information of persons with SID to their caregivers.

\subsection{Caregivers' Situation Awareness and Peripheral Display}

Caregivers usually work in a busy environment. The attention of caregivers has to be divided into multiple tasks which require varying types of information [30]. To respond sensitively towards a client's needs and wishes, caregivers need adequate situational awareness of their client's current condition, even while they are cooking or finishing the daily records on the computer $[6,30]$. Situation awareness refers to the correct perception of the state of an environment and system, and its predicted course [5]. Effective situation awareness helps caregivers to make decisions in their busy working environment. However, today's state-of-the-art monitoring systems usually interrupt an operator at inconvenient times and require focused attention, which results in added attention burden on caregivers $[8,13,18,26]$.

Peripheral displays provide an opportunity to convey clients' monitoring information in the periphery of caregiver's attention, eventually enhancing their situational awareness without added attention burden $[4,8,14,15]$. A peripheral display is an information display which targets different levels of attention by increasing or decreasing its saliency [12]. In other words, it can be a feasible technology to support timely follow-up of clients' pain conditions in a busy environment. For example, a peripheral display was designed for unpaid caregivers to remotely detect a client's in-home activity condition (real-time location, route, amount of time) [2]. A peripheral display with different visual, sound and haptic feedback in different scenarios was explored to assist caregivers monitoring sleep of dementia clients [4]. Davis and colleagues investigated the possibilities for subtle awareness in relation to social connectivity between the elderly and their caregivers [15]. Caregivers will quicker detect anomalies and have lower stress levels when they can keep constant tabs on their clients' well-being.

\subsection{Objectives}

The benefits of using a peripheral display to convey client information to caregivers have been shown in previous studies [2, 4, 8, 14]. Still, a successful development of a peripheral display for caregivers should take into account the specific usability context (e.g. what tasks or activities happen in a specific context, what type of information will/can the caregivers access), caregivers' mental model (e.g. how can display match certain patient's conditions in caregivers' mental representation) and task-specific cognition (e.g. how and when do caregivers make a particular decision) $[8,20]$.

To further define these requirements, the current study targets caregivers who work in care homes for persons with SID, and aims to understand the working context and perceptual process of these caregivers, and reflects the development of a peripheral display for assisting caregiver detecting real-time pain condition of persons with SID. As the main part of the proposed Pain App and also a part of a Bio response System, the peripheral display is expected to support caregivers' situation awareness and help with a sensibility to pain detection without adding attention burden.

\section{METHOD}

Overall, a user-centered design (UCD) process with iterative evaluation and redesign was implemented. The values of designing a care information display through a UCD approach have been shown in a systematic review and meta-analysis [20]. Target users (caregivers) and potential users (e.g. experts related to formal or informal care of SID clients) were involved in every iterative design and evaluation activity to ensure the comprehensive understanding of the user and context [7, 16, 21]. A schematic overview of the development process is shown in Figure 1.

\section{DESIGN PROCESS AND RESULTS}

\subsection{Participants}

The number of participants that contributed to the development process and their roles related to pain monitoring among persons with SID are shown in Table 1

\subsection{Exploration Stage}

A field trip to a care home of Bartiméus, an organization that provides care for persons with a visual or visual-and-intellectual disability, was taken to meet caregivers and visit their working place. To know the daily work especially the workflow when caregivers notice unexpected things happening on their clients, two experienced caregivers for clients with SID were interviewed in a semistructured way. Afterwards an expert conference was held with experts on the care for persons with SID (general practitioner for persons with SID, behavioral therapist, psychologist, researchers on pain behavior in persons with SID). The main findings in this stage can be summarized as three themes: existing workflow to identify anomalies in clients, different working scenarios, and types of information display. According to this analysis, design criteria were set.

3.2.1 Existing workflow to identify anomalies in clients. From the interviews, the current workflow to detect anomalies in clients' behavior was studied. Caregivers note changes in the state of their 
Table 1: Related information about the participants in each stage of the development process

\begin{tabular}{|c|c|c|}
\hline Stage & Participants (n) & Roles \\
\hline \multicolumn{3}{|l|}{ Exploration Stage } \\
\hline Caregiver Interviews & 2 & Caregivers for persons with SID \\
\hline Expert Group Meeting & 6 & $\begin{array}{l}\text { Doctor, Behavioral therapist, researcher, psychologist related to formal or } \\
\text { informal caring of SID clients }\end{array}$ \\
\hline \multicolumn{3}{|l|}{ First Iteration } \\
\hline Mood Board & 4 & $\begin{array}{l}\text { Caregiver, psychologist, researcher related to formal or informal caring of SID } \\
\text { clients }\end{array}$ \\
\hline User \& Expert Evaluation & 4 & $\begin{array}{l}\text { Caregiver, psychologist, researcher related to formal or informal caring of SID } \\
\text { clients }\end{array}$ \\
\hline Desktop Walkthrough & 1 & Psychologist who used to be a caregiver for persons with SID \\
\hline \multicolumn{3}{|l|}{ Second Iteration } \\
\hline User Evaluation in field & 2 & Caregivers for persons with SID \\
\hline
\end{tabular}

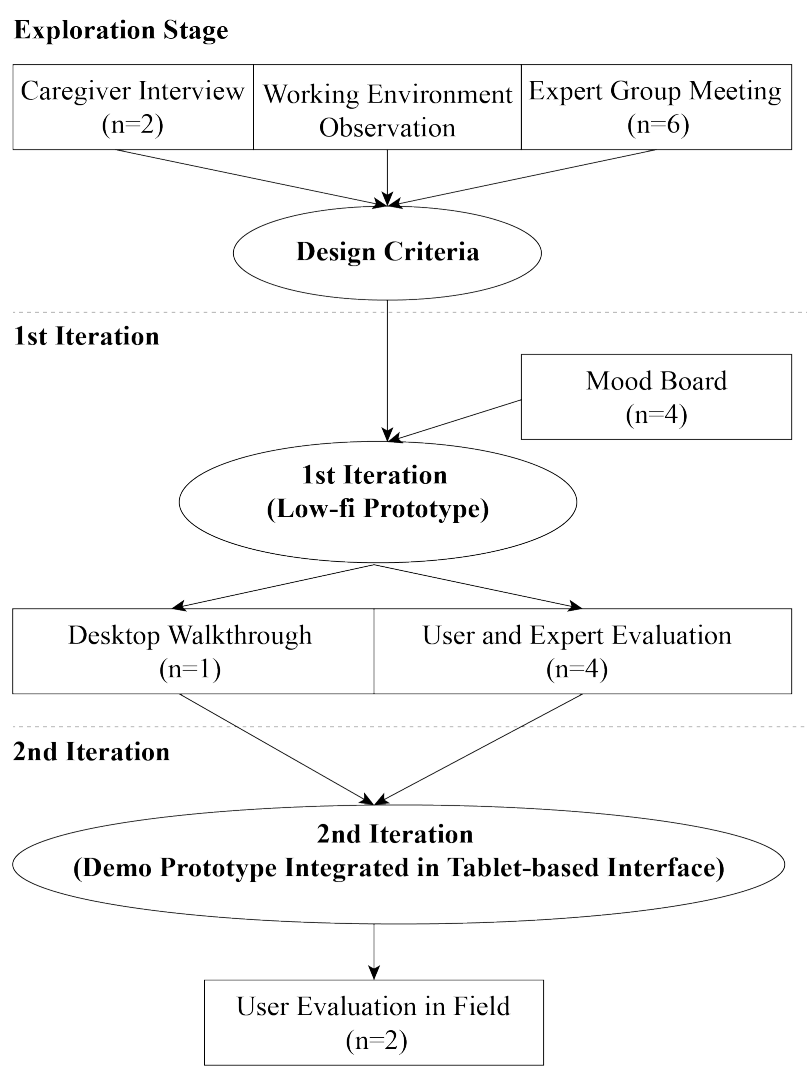

Figure 1: Schematic overview of the development of a Peripheral Display for a future Pain App

clients with SID through facial expression or abnormal behavior (i.e. gasp for breath, hit themselves). Not only from observing, these signals sometimes also come from clients' verbalizations or through the sound monitoring device as shown in Figure 2.

Caregivers will go to a client when receiving these visual or audio signals, though they normally do not know the level of distress (or pain) due to limited cues. Usually, they will first try to calm

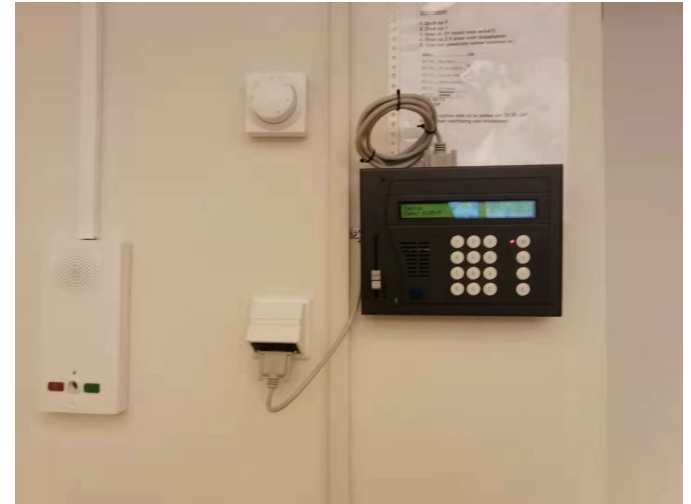

Figure 2: Sound monitoring device in care home

the client down, then offer help depending on their perception of the state of the client. Afterwards in the current procedure, the caregiver makes a record of this incident, by including the behavior of the client and the responding solution in the client's digital profile which can be reviewed by other caregivers of this client, parents, the physical therapist and the general practitioner, etc.

- Scenario 1: The caregiver interacts with a client; e.g. plays a game in the common space or provides hygienic care.

- Scenario 2: The caregiver works on a computer; e.g. making a daily report of behavior and activities or has night watch.

- Scenario 3: The caregiver carries out domestic activities and cannot directly access information on a client's state from a computer or table interface; e.g. preparing breakfast.

Moreover, two or three caregivers with different functions usually work in a care home for multiple clients. The varied functions of caregivers' create their own working scenarios. For instance, an assistant or intern usually remains with the clients in the common area (Figure 3 (Left)), while the head caregivers work on the computer (Figure 3 (Right)) or walks around to perform other tasks.

3.2.2 Types of information display. Types of visual, audio and haptic displays to convey pain information of clients to their caregivers have been discussed in the caregiver interviews and the expert 


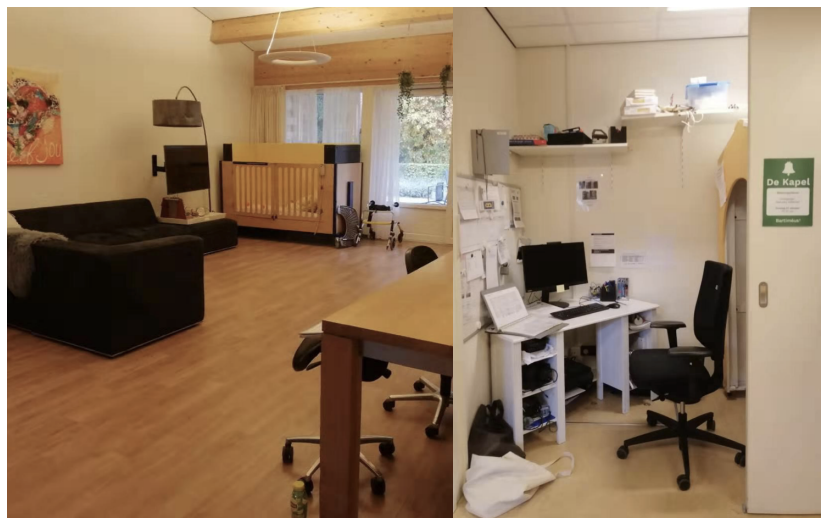

Figure 3: (Left) Common area for caregivers and clients; (Right) Caregivers' working space

group meeting. Almost all the participants were concerned about the audio display.

"The care home is usually noisy when many clients are in the common area, especially when a client listens to music in the music room." - an experienced caregiver.

"There is a previous study that found that clients with SID will behave in specific ways on purpose if they find this behavior could trigger certain sounds or changes" - a behavioral therapist in the field of pain related behavior of persons with SID.

Visual and haptic display seem to be more feasible in this context. Haptic displays allow for higher privacy of information conveying and less visual distraction, but give limited information. A visual display can cause a higher visual burden but display more adequate information. These pros and cons are kept in mind when designing.

3.2.3 Design criteria. Depending on the principle of peripheral displays [29] and findings from interviews, observations and an expert group meeting mentioned before, the design criteria for the peripheral display assisting caregivers in monitoring clients' real-time pain condition were summarized as follows:

Awareness As illustrated in Figure 4, a display should allow caregivers' situation awareness through different levels of peripheral attention (divided or inattention) [29].

Appeal The display should give relevant information on "pain" but cannot add (much) extra stress to caregivers.

Learnability The display should be easy to understand.

Effect of Breakdown The breakdown of monitoring system should be able to notice from the display.

Distraction The display cannot distract the caregiver from a primary activity while clients' conditions are normative.

\subsection{First Iteration}

To inspire the first iteration concept, a mood board was used to study users' mental models about perceiving "pain" from visual elements. Nine pictures (shown in Figure 5) expected to be perceived as different levels of pain and different levels of saliency were searched online. Four participants including caregivers and research

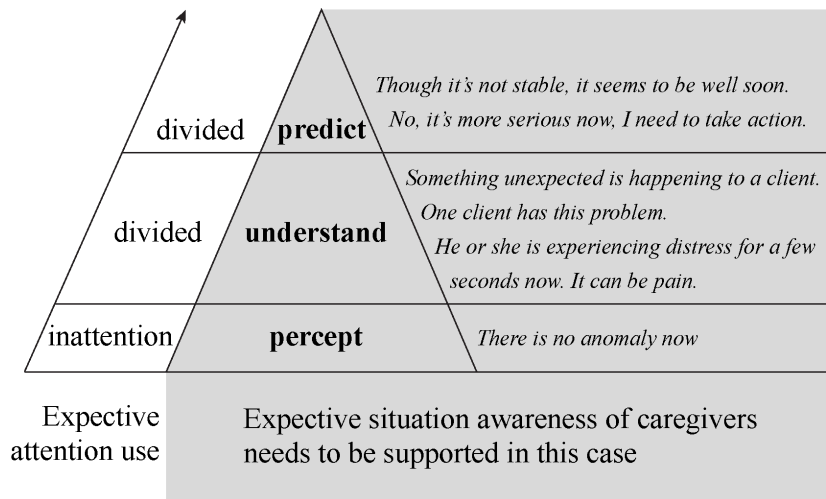

Figure 4: The hierarchy of caregivers' situation awareness should be supported by the display's design through different levels of peripheral attention (divided/inattention)

experts were asked to rate all pictures and place them in a graph on the mood board, where the $\mathrm{x}$-axis went from comfortable to pain and the y-axis from easy to attract attention to difficult to attract attention. The result of this expert rating can be viewed in Figure 5. Depending on design criteria and research findings, as well as inspiration from the mood board, several design concepts with haptic or visual displays for different scenarios were developed in the first iteration (see Table 2). These concepts were illustrated by a combination of text descriptions and low-fidelity animation prototypes to participants.

In the user and expert evaluation, four participants were asked to review these concepts from the first iteration with the method of thinking aloud. Participants were asked to speak out or write down anything they initially thought. Hereafter, participants were interviewed in a semi-structured way to collect subjective feedback on each concept.

It is worth to mention that the discussion of the first iteration concept in the user and expert evaluations were based on a hidden precondition that caregivers can successfully access the display. Whether these displays can indeed be easily accessed in specific daily scenarios remains questionable. A Desktop Walkthrough was held in order to have a more comprehensive understanding about caregivers' needs for the system during the day. For example, when do caregivers need feedback? What type of information carrier is possible to use in specific scenarios throughout the day? In this session, the paper toolkit (see Figure 6), which includes a simple map of a care house, cards representing caregivers, different times of day and three different devices (a tablet to provide visual displays, a smart wrist band to mainly provide haptic feedback and limited visual information, and a smartphone which is portable and allows both haptics and visual displays), was introduced to caregivers. After giving a time of day, the participant was asked to place caregiver cards on map-locations. Then the tasks of caregivers in that specific context would be discussed (e.g. which device do you think is feasible to use in this condition?).

3.3.1 Feedback on the haptic display concept. Feedback from user \& expert evaluation sessions and the Desktop Walkthrough to the "haptic" form was positive. A haptic feedback form can help convey 


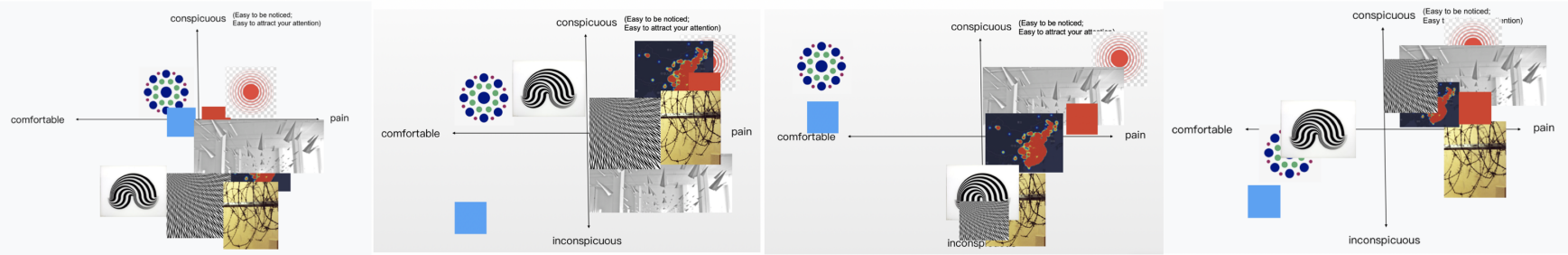

Figure 5: Mood board results from one caregiver, one psychologist and two other researchers related to formal or informal caring of SID clients

Table 2: Peripheral Display Concepts in the first iteration.

\begin{tabular}{ll}
\hline Concept & Description \\
$\begin{array}{l}\text { Haptic display } \\
\text { vibration }\end{array}$ & $\begin{array}{l}\text { When the pain possibility reaches a pre-set threshold, the wearable/portable d } \\
\text { vibrates. }\end{array}$ \\
$\begin{array}{l}\text { Wisual display } \\
\text { 1-black \& red }\end{array}$ & $\begin{array}{l}\text { of convexity and thickness of the lines increase, as well as the number of } \\
\text { (n) }\end{array}$
\end{tabular}

Visual display 2-colors \& red

A new dot appears in each interval to represent the mean value of possible pain during that interval. The dot appears closer to the center if the pain possibility is higher. When the dots in the center area reach a certain number, the shape morphs to a 'needle-shape'. Meanwhile, the oldest dot disappears with each new interval.
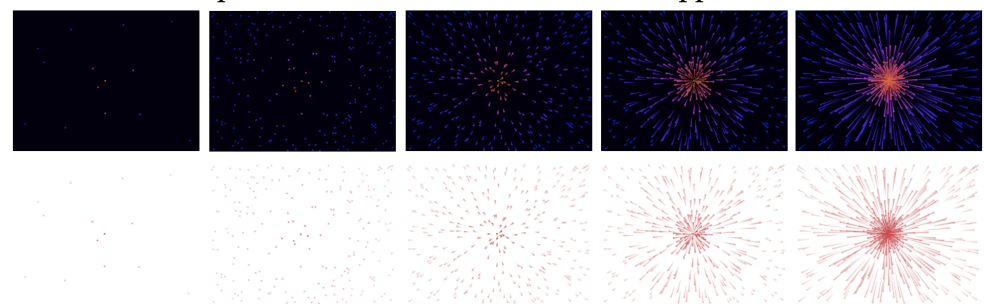

Visual display When the mean value of possible pain in a short interval increases, the number of 3

\section{Scenario}

Scenario 1 and 3 in which caregivers cannot always access visual information

Visual concepts are designed for Scenario 2, in which caregivers can always access visual information information when caregivers cannot access a fixed visual interface (in scenarios $1 \& 3$ ), though participants found the existing design unspecific. The limited information conveyed by the haptic display was indicated, by mentioning that the haptic feedback would not tell caregivers which client is in distress or provide them with information on what action to take.
A solution to combine a haptic alarm with a wearable screen such as a Smartwatch is suggested from the Desktop Walkthrough. Compared to a mobile phone, which has to be retrieved to receive information through the visual display, a wearable device with a screen like a Smartwatch is more convenient for caregivers who multitask in scenarios 1 and 3. 


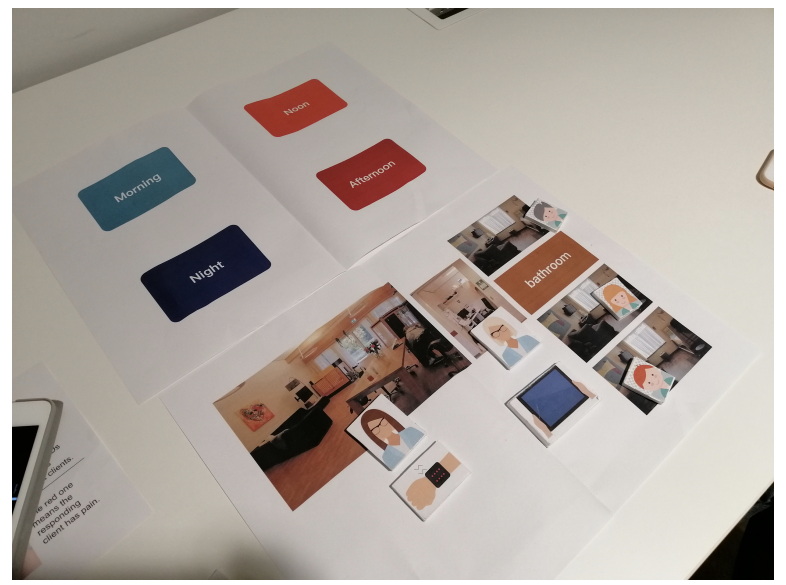

Figure 6: Paper toolkit used in the Desktop Walkthrough

3.3.2 Feedback on the visual display concepts. Feedback on the visual display concepts mainly came from the user and expert evaluation sessions. According to the mood board results, all participants could hardly associate concept 1 with "pain", although its "sharp angle" could be a "pain element" (Table 2). Moreover, two participants said they could not decide when to take action from viewing the display. In other words, users found the boundary between pain and no pain presented by concept 1 blurry.

Most participants agreed that visual display concept 2 was most relevant to "pain", though its many variables also made it difficult to understand. Moreover, the rules of this visual display were found complicated, which adds a mental burden on caregivers to 'read' the information. Concept 3 was deemed simple to read and easy to understand. The emerging feature did remind users of alertness and not only "pain". It helped to attract caregivers' attention, while it also could arouse unexpected stress.

In terms of color, two participants showed concerns on the stress caused by the color red, but another participant thought using red helped users to understand the meaning of the signal. The multi-color scheme of concept two received relatively more positive comments.

\subsection{Second Iteration}

Depending on the first iteration results, the visual display has been confirmed useful in all scenarios with different devices. Concept two of the visual displays was chosen to be simplified and improved. The number of dots in the display was fixed in any condition. Depending on real-time pain monitoring, the only dynamic part of the visual display is the length of the dot. When during real-time monitoring the pain probability reaches a set threshold, the dots start to lengthen.

As shown in Figure 7, a web-based prototype demonstration presented on a tablet was developed to test the second iteration concept. Because the current Bio response System cannot read pain information from detected skin conductance variability, a synthetic dataset representing fake pain conditions during a period of time was used as input to simulate the generative visual display. Processing was used to process the data and generate a dynamic visual display.

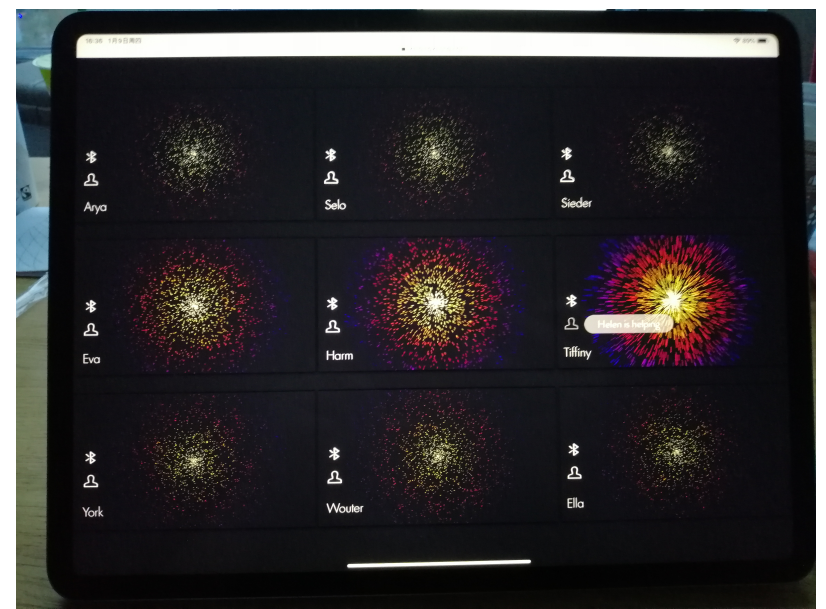

Figure 7: Demo prototype integrated into tablet-based interface

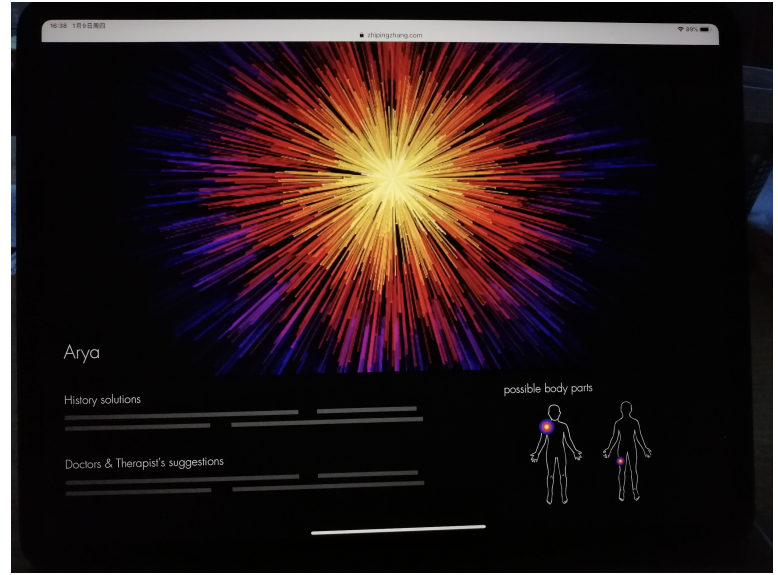

Figure 8: A page displaying conditional suggestions to explore possibilities of additional information on caregivers' processes

Videos recorded from the dynamic visual displays were integrated into a website to be used on a tablet.

Returning to the expected situation awareness of caregivers as shown in Figure 4, the design described was mainly of use for the "percept" and "understand" aspects of the process. According to feedback given by several caregivers in an earlier study on the arousal app [17], additional information shown in an app is expected to help "predict" decision-making processes of caregivers in response to clients' pain. Although caregivers might notice the changing pattern in their periphery and predict or decide based on the changing trend, additional information might further help them. A research-through-Design method with a prototype shown in Figure 8 was used to explore which information can support a caregivers' decision-making process.

The second iteration prototype was evaluated in the field, because the human attention span and shifting of attention are subtle things 


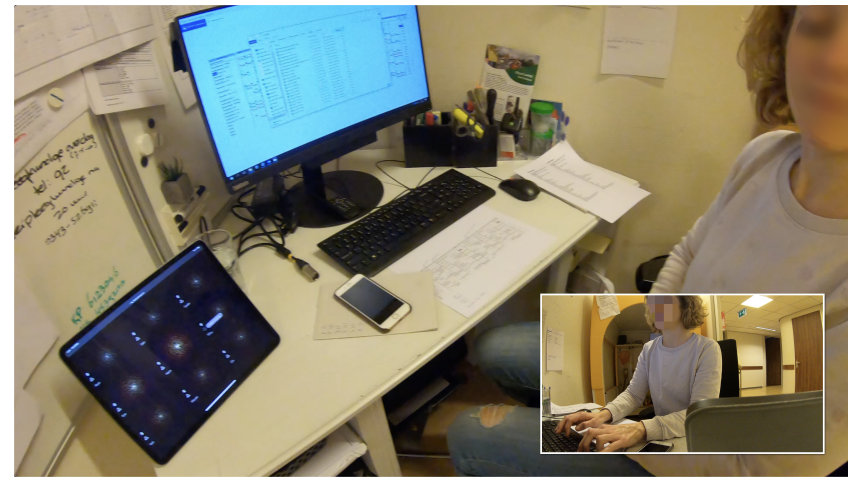

Figure 9: Field user evaluation setting for the $2^{\text {nd }}$ iteration

to be recognized and remembered. The context of scenario 2, working on a computer, was chosen for the test. The evaluation setting is illustrated in Figure 9. Two caregivers were respectively asked to use the design for fifteen minutes while they were doing administrative work. During this fifteen minutes, there will be fifteen pain moments from "Tiffny", indicated by the display as shown in Figure 7. After that, semi-structured interviews based on the peripheral display evaluation were conducted to see how the design fit with the criteria defined in the exploration stage [29]. Videos were recorded to ensure caregivers' responses could be reviewed by researchers (e.g. how often did the participants notice that pain occurred).

3.4.1 Feedback on the second iteration visual display concepts. Awareness The participants gave varied feedback on this aspect. One caregiver could sensitively detect the pain condition from the display. Shown on the video, her shift in attention was nearly perfect, as the designer expected that "...the display was always within my periphery while I worked on the other screen." The other caregiver could detect pain only four time (4/15), which was limitedly sensitive. She said "Most of the time, the display was out of my sight. Only when big changes were shown could I notice it". Fortunately, reasons to shift attention to the visual display were the same. Both of the participants mentioned that they noticed some changes with my peripheral vision. This indicates that peripheral vision differed between subjects. Preferably, the amplitude of variation in the display should be able to be adjusted by the users themselves. In terms of the additional information to support caregiver decision-making, caregivers showed neutral attitudes towards the information on pain locations, which at the moment cannot be measured. One participant thought integrated real-time video- or audio-monitoring material would be helpful, because they normally use this to support a subsequent action. Another participant thought guidelines with detailed steps to guide action for calming down or helping clients would be useful.

Appeal The display really reminded caregiver of "pain" and even different levels of pain possibility, while they did not experience added stress. As one of the participants said that "No, I don't feel stress. The feeling was neutral, just knowing something happened". Learnability All participants found the design easy to understand, after only a short explanation was offered.
Distraction The display was not found to be too distracting for participants.

\section{DISCUSSION}

In the current study it has been attempted to create a peripheral visual display of pain experience to be used by caregivers for persons with severe intellectual disability (SID), in order to support them in clients' pain detection when doing desk work. During the design process caregivers' mental model and task-specific cognition has been taken into account, as well as their specific thoughts and needs while performing daily tasks. The study has resulted in a visual display that has been shown to attract the caregiver's attention when needed but does not distract from a computer task or generate a stress response.

Regarding the exploration stage it was discovered that caregivers receive subtle and limited cues from clients about their state of well-being and the caregivers' response is based on incomplete information of their client's level of distress or pain. Caregivers provided three separate working scenarios, where they would benefit from additional haptic or visual information on their client's well-being. In the first iteration of the design process, caregivers and expert researchers provided data on their own mental model regarding pain and judged three types of designs on their usability in daily caregiving for persons with SID. This has provided valuable knowledge on the thoughts and needs of a unique target group regarding a quite complex concept (pain). In the second iteration of the design process an improved design was tested in a work environment and the responses were mostly positive. The design was found to be uncomplicated and would only attract attention on the necessary moments, while caregivers found they could remain focused on their other task when the display did not indicate pain. This result indicates that a well-designed peripheral display of pain could aid caregivers to respond more effectively to their client's pain and distress.

The data gathered in this study contributes to existing knowledge with information on a niche user group (caregivers of persons with SID living in care homes). As mentioned before, research on this target group has been scarce, while these caregivers spend their days caring for persons for whom communication and expression is difficult or impossible. Challenges and stresses the caregivers face during their work are plentiful and in this study we created part of an aid in pain recognition with the goal of combating one of the challenges and reducing some of the stress.

\section{LIMITATIONS AND FUTURE GUIDELINES}

Feedback from the user in-site evaluations indicated that the peripheral display design from this project has the potential to support caregivers in clients' pain detection in the context of performing desk work. Multi-factors researched and considered during the design process could be referred by similar studies about peripheral display, especially the studies in the semi-hospitalized contexts. However, considering the short project time, there are still several limitations on the process and outcomes. 


\subsection{Evaluation methods}

Throughout the project only qualitative evaluations were gathered. Deep and subjective results that come from qualitative evaluation can help push the design iteration and improvement, however, to test whether the design fits with design criteria or certain objectives, quantitative evaluations can provide more objective evidence. Therefore, a quantitative evaluation on the display concept was performed in a further study [10].

\subsection{Usability scenarios}

The existing design outcome only fits within the context of desk work (scenario 2). Although the head caregiver's time is mostly spent there, many other working scenarios should be taken into account. The results from the Desktop Walkthrough have already indicated the potential use of wearable devices with a visual screen and vibration module, when the caregiver cannot access a fixed visual screen. In further development, this combination should be evaluated by caregivers on-site. Moreover, considering that caregiving equals teamwork, cooperation between caregivers in a care home should also be supported by the system.

\subsection{Multi-device experience}

Results from the research and evaluations suggest the need for different devices to meet various needs and constraints in different working contexts. In further development, the question of how to keep a multi-device caregiver experience consistent, continuous and/or complementary to pain detection should be answered. Furthermore, whether an adjusted Bio response System helps caregivers detect pain in clients with SID effectively and adequately will need to be further researched.

\section{CONCLUSION}

This project focused on developing an information display for a Pain App, as a part of a caregiver pain detection system for persons with SID. Throughout the user-centered design process, understanding of the mental model and working context of caregivers was constantly expanded. To develop a peripheral display which supports caregivers' situation awareness and help with sensibility to pain detection without attention burden, multi-factors about caregivers and their clients were considered based on the principle of peripheral displays [29]. Regarding the risk of clients' reaction to certain sound and the working environment is noisy, audio feedback was not included in the display concept. Considering that the working scenarios of multi-users (caregivers) are fixed in a space at the same time, Desktop Walkthrough and field user test were conducted to evaluate two iterations of the display concepts. The final user evaluations showed that a peripheral display design can support caregivers' situation awareness and timely response to a client's pain condition. However, the result also indicated that peripheral vision differed between subjects. Preferably, the amplitude of variation in the display should be able to be personalized. The user study and design outcomes of this project are expected to enrich the peripheral display applications in similar semi-hospitalized contexts.

\section{REFERENCES}

[1] A.C. Günther et al. 2016. Pain rather than induced emotions and ICU sound increases skin conductance variability in healthy volunteers. Acta Anaesthesiologica Scandinavica 60, 8 (2016), 1111-1120. DOI:http://dx.doi.org/10.1111/aas.12751

[2] C.A. Wingrave, M. Rowe, and S. Greenstein. 2012. WIP: Designing Smart Systems to Support @Work Caregiver Needs. In Artificial Intelligence for gerontechnology: Papers from the AAAI Fall Symposium. Palo Alto, CA: AAAI Press.

[3] C.G. Janssen, C. Schuengel, and J. Stolk. 2002. Understanding challenging behaviour in people with severe and profound intellectual disability: A stressattachment model. Journal of Intellectual Disability Research 46, 6 (2002), 445-453. DOI:http://dx.doi.org/10.1046/j.1365-2788.2002.00430.x

[4] Carlos A. Alemán and Jesús Favela. 2016. Ambient displays to assist caregivers monitoring the sleep of people with dementia. Ubiquitous Computing and Ambient Intelligence (2016), 40-45. DOI:http://dx.doi.org/10.1007/978-3-319-48799-1_5

[5] Christian M. Schulz, Mica R. Endsley, Eberhard F. Kochs, Adrian W. Gelb, and Klaus J. Wagner. 2013. Situation awareness in Anesthesia. Anesthesiology 118, 3 (2013), 729-742. DOI:http://dx.doi.org/10.1097/aln.0b013e318280a40f

[6] David Howe. 2006. Disabled children, parent-child interaction and Attachment. Child Family Social Work 11, 2 (2006), 95-106. DOI:http://dx.doi.org/10.1111/j. 1365-2206.2006.00397.x

[7] Erik Hollnagel. 2010. Handbook of Cognitive Task Design, Boca Raton, Fla: CRC Press.

[8] Frank A. Drews and Dwayne R. Westenskow. 2006. The right picture is worth a thousand numbers: Data Displays in Anesthesia. Human Factors: The fournal of the Human Factors and Ergonomics Society 48, 1 (2006), 59-71. DOI:http://dx.doi. org/10.1518/001872006776412270

[9] Givaudan Foundation. Switzerland: Olfaction research to communicate with disabled. Retrieved May 9, 2021 from https://www.givaudan-foundation.org/ projects/switzerland-children

[10] Helen Kovring, P.S. Sterkenburg, Emilia Ivanova Barakova, and Loe M.G. Feijs. 2021. Designing pain visualisation for caregivers of people with special needs: A co-creation approach. Behaviour, Information and Technology (2021). in revisions.

[11] Huda Huijer Abu-Saad. 2000. Challenge of pain in the cognitively impaired. The Lancet 356, 9245 (2000), 1867-1868. DOI:http://dx.doi.org/10.1016/s01406736(00)03253-0

[12] Jennifer Mankoff, Anind K. Dey, Gary Hsieh, Julie Kientz, Scott Lederer, and Morgan Ames. 2003. Heuristic evaluation of ambient displays. Proceedings of the conference on Human factors in computing systems - CHI '03 (2003). DOI:http: //dx.doi.org/10.1145/642611.642642

[13] Joel E. Fischer, Chris Greenhalgh, and Steve Benford. 2011. Investigating episodes of mobile phone activity as indicators of opportune moments to deliver notifications. Proceedings of the 13th International Conference on Human Computer Interaction with Mobile Devices and Services - MobileHCI '11 (2011). DOI:http: //dx.doi.org/10.1145/2037373.2037402

[14] Juliane Pfarr, Michael T. Ganter, Donat R. Spahn, Christoph B. Noethiger, and David W. Tscholl. 2019. Avatar-based patient monitoring with Peripheral Vision: A Multicenter Comparative Eye-Tracking Study. Fournal of Medical Internet Research 21, 7 (2019). DOI:http://dx.doi.org/10.2196/13041

[15] Kadian Davis, Jun Hu, Loe Feijs, and Evans Owusu. 2015. Social Hue: A subtle awareness system for connecting the elderly and their caregivers. 2015 IEEE International Conference on Pervasive Computing and Communication Workshops (PerCom Workshops) (2015). DOI:http://dx.doi.org/10.1109/percomw.2015.7134015

[16] Karen Holtzblatt, Jessamyn Burns Wendell, and Shelley Wood. 2005. Rapid contextual design. Ubiquity 2005, March (2005), 3-3. DOI:http://dx.doi.org/10.1145/ 1066348.1066325

[17] Kyra Frederiks, Paula Sterkenburg, Emilia Barakova, and Loe Feijs. 2019. The effects of a bioresponse system on the joint attention behaviour of adults with visual and severe or profound intellectual disabilities and their affective mutuality with their caregivers. Journal of Applied Research in Intellectual Disabilities 32, 4 (2019), 890-900. DOI:http://dx.doi.org/10.1111/jar.12581

[18] Luis Leiva, Matthias Böhmer, Sven Gehring, and Antonio Krüger. 2012. Back to the App. Proceedings of the 14th international conference on Human-computer interaction with mobile devices and services - MobileHCI '12 (2012). DOI:http: //dx.doi.org/10.1145/2371574.2371617

[19] Lynn M. Breau, Carol S. Camfield, Patrick J. McGrath, and G.Allen Finley. 2003. The incidence of pain in children with severe cognitive impairments. Archives of Pediatrics \& Adolescent Medicine 157, 12 (2003), 1219. DOI:http://dx.doi.org/10. 1001/archpedi.157.12.1219

[20] Melanie C. Wright et al. 2019. Critical care information display approaches and design frameworks: A systematic review and meta-analysis. fournal of Biomedical Informatics 100 (2019), 100041. DOI:http://dx.doi.org/10.1016/j.yjbinx.2019.100041

[21] Mica R. Endsley and Debra G. Jones. 2012. Designing for situation awareness: An approach to user-centered design, London: Taylor \& Francis.

[22] P. Poppes, A.J.J. van der Putten, W.J. Post, and C. Vlaskamp. 2016. Risk factors associated with challenging behaviour in people with profound intellectual and multiple disabilities. Journal of Intellectual Disability Research 60, 6 (2016), 537552. DOI:http://dx.doi.org/10.1111/jir.12268 
[23] P. Stallard. 2001. Pain in cognitively impaired, non-communicating children Archives of Disease in Childhood 85, 6 (2001), 460-462. DOI:http://dx.doi.org/10. 1136/adc.85.6.460

[24] P.S. Sterkenburg, K. Frederiks, Emilia Ivanova Barakova, PJ.F. Peters, Loe M.G. Feijs, and W. Chen. 2017. A Bioresponse System for Caregivers of Adults with Severe or Profound Intellectual Disabilities. Fournal of Mental Health Research in Intellectual Disabilities 10, sup1 (2017), i-222. DOI:http://dx.doi.org/10.1080/ 19315864.2017.1368259

[25] Sandra M.G. Zwakhalen, Katinka A.J. van Dongen, Jan P.H. Hamers, and Huda Huijer Abu-Saad. 2004. Pain assessment in intellectually disabled people: Nonverbal indicators. Fournal of Advanced Nursing 45, 3 (2004), 236-245. DOI:http //dx.doi.org/10.1046/j.1365-2648.2003.02884.x

[26] Shamsi T. Iqbal and Eric Horvitz. 2010. Notifications and Awareness. Proceedings of the 2010 ACM conference on Computer supported cooperative work - CSCW' 10 (2010). DOI:http://dx.doi.org/10.1145/1718918.1718926
[27] Shobha Malviya, Terri Voepel-Lewis, Sandra Merkel, and Alan R. Tait. 2005. Difficult pain assessment and lack of clinician knowledge are ongoing barriers to effective pain management in children with cognitive impairment. Acute Pain 7, 1 (2005), 27-32. DOI:http://dx.doi.org/10.1016/j.acpain.2005.01.002

[28] Sien Vandesande, Guy Bosmans, Paula Sterkenburg, Carlo Schuengel, Wim Van Den Noortgate, and Bea Maes. 2019. Comfort provided by parents versus strangers after eliciting stress in children with severe or profound intellectual disabilities: Does it make a difference? Attachment \& Human Development 22, 4 (2019), 425447. DOI:http://dx.doi.org/10.1080/14616734.2019.1659835

[29] Tara Matthews, Tye Rattenbury, and Scott Carter. 2007. Defining, Designing, and Evaluating Peripheral Displays: An Analysis Using Activity Theory. HumanComputer Interaction 22, 1-2 (2007), 221-261. DOI:http://dx.doi.org/10.1080/ 07370020701307997

[30] Yunan Chen, Victor Ngo, and Sun Young Park. 2013. Caring for caregivers. Proceedings of the 2013 conference on Computer supported cooperative work-CSCW '13 (2013). DOI:http://dx.doi.org/10.1145/2441776.2441789 